\title{
6 Ethik und Erfolg
}

Welche Aspekte von Erfolg gibt es für ein Krankenhaus? Welchen Nutzen hat die Ethik dabei? Kann Ethik auch negative Auswirkungen auf den betriebswirtschaftlichen Erfolg haben? Könnten sich nur wirtschaftlich gesunde Krankenhäuser Ethik leisten oder trägt Ethik zur wirtschaftlichen Gesundung bei?

\subsection{Vom Nutzen des Ethik-Managements}

Schon der „Gründungsvater“ der Management-Wissenschaft Peter Drucker war der Überzeugung, dass „weiche Faktoren“ ebenso wichtig sind, wie die vielfach erwähnten „Zahlen, Daten und Fakten“, die den „harten“ Fakten zugeschrieben werden. Ethik-Management gehört zum Bereich der weichen Faktoren. Es ist ein Element der Unternehmenskultur, und als solches schwer in „harter Währung“ $z u$ bewerten.

Wenn sich Führungskräfte oder ganze Unternehmen dafür entscheiden, eine Ethik-Infrastruktur aufzubauen, ethische Kompetenz der Teams zu fördern, Wissen über Ethik zu vermitteln und die Qualität ihrer Produkte, Strukturen, Arbeitsprozesse und Umgangsweisen nach ethischen Maßstäben zu bewerten, dann liegt es nahe, die Frage nach dem tatsächlichen Nutzen zu stellen. Die Frage ist berechtigt, aber sie lässt sich nicht durch einfache Evaluationsverfahren beantworten. 
Arbeitspakete, „Meilensteine“ und Messverfahren für Erfolg oder Misserfolg, vorgetragen in „Power-Point-Vorträgen“ in Projektgruppensitzungen sind hier bestenfalls unwichtig, schlimmstenfalls können sie für die Förderung einer ethischen Unternehmenskultur sogar schädlich sein, wenn sie nicht in effektive Gesprächskulturen eingebunden sind. Gewonnenes Vertrauen bei der Bevölkerung, bei Patienten und bei den Mitarbeitern selbst lässt sich schwer direkt messen. Allenfalls indirekt können Erfolge mittels Skalen und Zahlen abgeschätzt werden, ohne jemals sicher zu sein, welche Faktoren nun tatsächlich wirksam waren.

Eine Unternehmenskultur, die von Vertrauen lebt und Vertrauen schafft, lebt von wertschätzenden Beziehungen und Verhaltensweisen. Immanuel Kant, bedeutendster Repräsentant der Pflichtenethik (Deontologie) hatte aus moralischen Gründen eine „Verzweckung“ von Menschen verurteilt (s. Kap. 2). Würde eine Unternehmensethik nur oder in erster Linie dem Zweck dienen, die Gewinne eines Unternehmens zu erhöhen, so würde sie dem Geist dieser Ethik direkt zuwiderlaufen. Ethik soll letztendlich dem guten Leben, im Krankenhaus einer guten Medizin um ihrer selbst willen dienen. Ihr Sinn besteht letztendlich darin, dass den Patienten gute medizinische Hilfe gebracht und der Bevölkerung die Sicherheit einer entsprechenden Versorgung im Bedarfsfalle gegeben wird. Diese Ziele sind als kritischer Maßstab an alle betriebswirtschaftlichen Strategien anzulegen. Die Kunst des Managements besteht dann darin, „Versorgungsqualität“ in diesem Sinne zu ermöglichen, unter bestmöglicher wirtschaftlicher Nutzung von Ressourcen.

\subsection{Vertrauen und Kommunikation}

Da die Unternehmenskultur wesentlich durch die Haltung von Führungskräften und deren Umgang mit ihren Anvertrauten geprägt ist, sind auch die Kommunikationsweisen hinsichtlich ihrer moralischen „Impacts“ zu beachten. Monologisierende Führungskräfte stehen häufig versteinerten Zuhörern gegenüber, die sich stumm ihren Teil denken und zu keinerlei Compliance bereit sind. Stützt sich die Unternehmenskommunikation überwiegend auf die zur Regel gewordenen „Power-Point-Präsentationen“, in denen man gemeinsam gegen die Wand schaut (und sich nicht gegenseitig ins Gesicht), dann dürfte es schwerer sein, die gewünschte Kultur aufzubauen. Es liegt in diesem Modell eine eindimensionale Linearität vor, anstatt dialogisch zu kommunizieren und damit achtsam der Person des Gegenübers zu begegnen. Die Überlegenheit wertschätzender, offener und dialogischer Kommunikation gegenüber monologischer Top-Down-Beziehung in Hinblick auf Zusammenarbeit und Motivation muss nicht erst durch Messverfahren belegt werden. 
Der Unternehmensberater Reinhard Sprenger nennt als Kernkompetenz einer Führungskraft deren Potenzial „die Herzen der Menschen“ zu erreichen. Ohne Achtsamkeit kann das nicht gelingen.

„Entrümpeln, ausmisten, was Vertrauen und Anstand zerstört. Zum Beispiel Befragungen, Rankings, Boni und Incentives. Dann ist Würde kein Konjunktiv mehr. [...]

Führung ist eine kraftvolle Mischung aus Strategie plus Vertrauen. Aber wenn man auf eines verzichten muss, dann auf die Strategie. Ohne Vertrauen der Anvertrauten gibt es keine Führung. Wer hingegen die Herzen der Menschen nicht erreicht, hat in diesem Job keine Existenzberechtigung. Der ist nur Vorgesetzter." (Reinhard u. Vasek 2015)

Kann man messen, ob es den Führungskräften gelungen ist, die Herzen ihrer Anvertrauten zu erreichen? Würde nicht bereits der Versuch, dieses zu tun, zynisches Abwehrverhalten provozieren? Und selbst wenn es gemessen (besser: vermessen) wäre, was wäre das Ergebnis wert? Könnte nicht ein einziges Ereignis, vielleicht sogar schon die Veröffentlichung der Erfolgsmitteilung selbst, die Ergebnisse komplett verändern?

\subsection{Ethik und Unternehmenskultur als Erfolgsfaktoren}

In den 1990er Jahren empfahlen die Bertelsmann Stiftung und die HansBöckler-Stiftung auf Basis eines gemeinsam durchgeführten Forschungsprojekts den Unternehmen der deutschen Wirtschaft die sorgfältige Pflege einer „Unternehmenskultur als Erfolgsfaktor“. Die entsprechenden Veröffentlichungen waren überzeugend und sie wurden auch im Gesundheitswesen beachtet (s. Kap. 7). Gleichwohl beruhte die Überzeugung auf der Kraft der Argumentation, nicht auf Messwerten. Andere Studien, insbesondere aus den USA, untermauern die Bedeutung „ethischer Infrastrukturen“ für die Stärkung „ethischen Verhaltens“. Unternehmenserfolge gemessen in wirtschaftlichen Daten wurden jedoch nicht systematisch erforscht.

Positive Effekte von Maßnahmen zur Stärkung ethischer Bewusstheit und Kompetenz in Krankenhäusern (am Krankenbett und im Management) sind auf verschiedene Weise zu erwarten. Im Vordergrund stehen medizinische Effekte wie bessere Indikationsstellungen und Therapieentscheidungen, insbesondere bei der Intensiv- und Palliativmedizin, aber auch in der Psychiatrie oder der Kinderheilkunde. Konflikte innerhalb der Teams oder mit Angehörigen können so vermindert werden. Damit werden die Patientenorientierung und die Versorgungsqualität gestärkt. An- 
gesichts deutlich wachsender Zahlen von Patienten sehr unterschiedlicher kultureller Herkunft können so Entscheidungshemmnisse und Konfliktpotenziale besser beseitigt oder jedenfalls vermindert werden. Positive Einflüsse auf die Unternehmenskultur ergeben eine Verbesserung der Mitarbeiter-und Patientenzufriedenheit, die auch von externen Stakeholdern wahrgenommen werden wird.

\subsection{Verbesserung der medizinischen Qualität}

Wichtige Qualitätsaspekte der medizinischen Versorgung sind ethischer Natur. Die subjektiv empfundene Würde des Menschen und deren Missachtung oder Verletzung spielt eine entscheidende Rolle bei der Beurteilung einer Klinik und bei der Frage, ob diese erneut von Patienten aufgesucht wird oder nicht. Respektloses Verhalten des Personals bedeutet schlechte Versorgungsqualität. Die Beachtung der Autonomie des Patienten, seiner Selbstbestimmung und seiner Rechte als Patient (gleichwohl der Menschenrechte!) ist von größter Bedeutung für Patienten, Angehörige, die Öffentlichkeit und für das Klima innerhalb der Behandlungsteams selbst. Hierzu muss nicht erst ein methodisch aufwändiges und vom Ergebnis her zweifelhaftes Messverfahren zum Einsatz kommen um den Wert solcher Umgangsformen und Entscheidungswege für Patienten und Klinikum anzuerkennen.

Die therapeutischen Berufe entscheiden und handeln besser und bewusster in Bezug auf die Belange ihrer Patienten. Die medizinischen Entscheidungsprozesse werden klarer hinsichtlich ihrer Kriterien und hinsichtlich der Beteiligten und ihrer Rolle. Dies gilt nicht nur für so genannte „Endof-Life-Decisions“, die ja keine mehr sind, wenn der Patient auf Grund der medizinischen Maßnahme überlebt. Untersuchungen zeigen, dass die Kenntnis der „Principles of Biomedical Ethics“ (s. Kap. 2) dazu führt, dass schwierige medizinische Entscheidungen wesentlich schneller, strukturierter und konfliktärmer getroffen werden können.

\subsection{Beitrag zur Patientenorientierung und Qualitätssicherung}

Ein Krankenhausunternehmen, das es wirklich ernst meint mit dem Anspruch konsequenter Patientenorientierung, braucht keinen empirischen Nachweis, dass diese ein betriebswirtschaftlich relevantes Ziel ist. Es ist klar, dass ein Krankenhausteam, bestehend aus Management, Pflege, Ärzten und weiteren Berufsgruppen (s. Kap. 2), das seine Entscheidungen und Handlungen auf die Ziele und Bedürfnisse jedes einzelnen Patienten abstimmt, damit einen Beitrag zu einem „guten Krankenhaus“ liefert. 
Ebenso einleuchtend ist es, dass Verletzungen ethischer Crundsätze niemandem nutzen und dass diese immer wieder mit Sensibilität aufzuspüren und abzustellen sind.

Eine Ethik-Infrastruktur, formell und informell, entfaltet ihre Wirksamkeit nach zwei Seiten. Zum einen fördert sie die Qualität der Patientenbetreuung. Das betrifft die medizinischen Entscheidungen, das Engagement der Teams, die Aufmerksamkeit der Kliniker für die Belange der Patienten und der Angehörigen. Zum anderen fungiert sie als eine Art Radar, das problematische Entwicklungen erfasst, zur Sprache bringt und so weit wie möglich beseitigt. Damit leistet sie einen wichtigen Beitrag zur Qualitätssicherung, der gegenüber der Auswertung von Patientenoder Angehörigenbeschwerden durch das Qualitätsmanagement schneller und sensibler ist.

\section{Patientenorientierung und Ethik in der Praxis}

Bericht eines Angehörigen als Rundmail an Freunde und Verwandte über die Betreuung seines Bruders auf einer Intensivstation, deren Ärzte und Pflegekräfte ethisch geschult sind. Der Bruder des Patienten war als Betreuer eingesetzt. Er war dankbar, dass nicht er selbst die Entscheidung zur Änderung des Therapieziels treffen musste.

„Wir hatten gestern Mittag noch zwei Gespräche mit den Ärzten und sie haben uns beide klargemacht, dass es für Herbert keine Hoffnung gibt. Der Zustand seiner Lunge ist so schlecht, dass die günstigste Prognose, die an sich schon sehr unwahrscheinlich ist, ein Leben in einem Pflegeheim mit ständiger Beatmung ist.

Wir haben den Ärzten dann auf Nachfrage gesagt, dass Herbert das alles sicher nicht wollen würde. Daraufhin haben die Ärzte (nicht wir!) entschieden, sich aus der Behandlung zurückzuziehen. Das Therapieziel (so nennt sich das in der Fachsprache) wurde daraufhin geändert, und war nun nicht mehr Herberts Genesung, sondern, dass er ruhig einschlafen kann. Dafür wurden nicht die Maschinen abgeschaltet (was ja eine weit verbreitete Meinung ist), sondern seine Medikamente anders dosiert.

Wir (meine Eltern, Doris und ich) waren dann dabei. Eine Schwester und ein Arzt waren auch im Zimmer, hielten sich aber im Hintergrund auf. Herbert hat - so ist zumindest unser Eindruck - nicht mehr gelitten. Er ist dann friedlich eingeschlafen. Es war sehr schwer, dabei zu sein, aber auch gut, sich so zu verabschieden.

Ich kann euch versichern, dass die Ärzte in den vier Wochen von Herberts Aufenthalt wirklich alles getan haben, was möglich war. Es wurde nichts unversucht gelassen, und man hat in den Gesprächen gestern gemerkt, dass es auch den Ärzten schwerfiel, Herbert gehen zu lassen. 


\begin{abstract}
Überhaupt kann man sagen, dass sowohl Herbert als auch wir Angehörige vom gesamten Team der Station super betreut wurden. Herbert wurde immer respektvoll behandelt, man hat ihm immer gesagt, wenn etwas mit ihm gemacht wurde („Herr H., ich werde Sie jetzt auf die Seite drehen“, etc.) auch wenn er das wahrscheinlich gar nicht gehört hat. Auch unsere Fragen und Ängste wurden sowohl von den Pflegern als auch von den Ärzten immer ernst genommen. Man hat sich Zeit genommen, und uns auch außerhalb der Besuchszeiten zu Herbert gelassen. Ich schreibe das deshalb, weil es ein gutes Gefühl ist, zu wissen, dass Herbert in den letzten Wochen seines Lebens in guten Händen war.

(Die Namen wurden geändert.)
\end{abstract}

Die positiven Effekte einer Ethik-Infrastruktur und eines Ethik-Managements lassen sich nicht sicher messen, allenfalls werden sie in Berichten wie dem obigen erkennbar. Freilich gibt es auch ähnlich positive Versorgungsbeispiele ohne dass eine explizite Ethik-Integration vorgenommen wurde. Dennoch spricht die Erfahrung dafür, dass ethische Bewusstheit im Krankenhaus den Patienten und ihren Angehörigen besonders wertvolle Dienste leistet und den Behandlungsteams selbst eine höhere Arbeitszufriedenheit schafft.

\title{
Ethik gehört zum Kern des Qualitätsversprechens eines Krankenhauses, lässt sich aber noch schlechter über Kennzahlen abbilden als andere Qualitätsaspekte.
}

$\mathrm{Zu}$ den positiven Seiten gehört auch die Erfassung von unerwünschten Ereignissen, die eben nicht verschwiegen und einfach hingenommen werden, sondern von aufmerksamen und engagierten Mitarbeitern registriert und angesprochen werden. Ein Großteil der in Ethik-Cafés, Sprechstunden oder Ethik-Problemerhebungen erfassten Themen sind nicht die Wertekonflikte, die für manche Philosophen und Theologen als Bedingung für eine ethische Fallbesprechung gelten. Mehrheitlich handelt es sich um problematische Handlungen, Entscheidungen oder Strukturen, die als Verletzung medizin- und pflegethischer Grundsätze gelten. Es kommt also nicht darauf an, ob im akademischen Sinne ein Ethikkonflikt vorliegt, sondern es geht um „gute Medizin und Pflege“ und um „gute Führung, gutes Management.“ 


\section{Praxisbeispiele für Problembearbeitungen in der Ethik-Infrastruktur}

- Eine Schwester berichtet von Kollegen, die Patienten entwürdigend behandeln. Es wird ein Gespräch unter Einbeziehung der Pflegedirektion durchgeführt um Wiederholungen solcher Ereignisse zu vermeiden.

- Mitarbeiter berichten, dass mehrfach Sterbenskranke ihre letzten Tage im Badezimmer einer Station verbracht hätten. Das Klinische Ethik-Komitee lädt die Stationsmitarbeiter ein. Es wird deutlich, dass das Team Stellenstreichungen befürchtet, wenn ein Patient alleine im Zimmer wäre und die anderen Betten gesperrt würden. Gemeinsam mit dem Patientenmanagement wird eine Lösung erarbeitet, um diese Sorge zu nehmen. Die Direktion erklärt, dass Sterbende ohne Nachteil für die Stationen in ihren Zimmern bleiben sollen.

- Dem klinischen Ethik-Komitee wird berichtet, dass Angehörige keine ausreichende Gelegenheit erhalten, sich von ihren Verstorbenen würdig zu verabschieden. Das Management ändert die Verträge mit einem Bestattungsunternehmen um den Missstand abzustellen.

- Physiotherapeuten unter Zeitdruck betreuen nur „coole Patienten“ und lassen Schwerstkranke aus. Ethik-Berater machen den Mitarbeitern klar, dass sie unbewusst „priorisieren“ und dass die dabei verwendeten Kriterien transparent und abgestimmt sein müssen. Mit dem Vorstand des Klinikums werden direkte Gespräche organisiert, um die Belastung der Therapeuten sowie die negativen Folgen für die Patienten zu verdeutlichen. Es wird eine einvernehmliche Lösung gefunden.

- Vorgesetzte ignorieren grundlegende Hygienemaßnahmen. Die Leitung des Ethik-Cafés meldet dies der zuständigen übergeordneten Führungskraft, die mit der betreffenden Person ein verwarnendes Gespräch führt. Zugleich wird diese davor gewarnt, den „Whistleblower“ zu benachteiligen.

- Ein Vorgesetzter schikaniert seit Monaten Mitarbeiter und Patienten. Zwei Pfleger berichten davon in der Ethik-Sprechstunde. Die gemeinsam mit der Gesamtleitung des Hauses eingeleitete Untersuchung der Fälle lässt erkennen, dass der Vorgesetzte an einer manifesten Schizophrenie leidet. Es gelingt, den Betroffenen einer Behandlung zuzuführen.

- Im Klinischen Ethik-Komitee wird berichtet, dass technische Mitarbeiter einer IT-Firma, die Computersysteme einrichtet, vertrauliche Patientenakten lesen. Der Vorstand wird alarmiert. Umgehend werden mit den Verantwortlichen der Firma Maßnahmen vereinbart, um Verletzungen des Patientengeheimnisses zu unterbinden.

All dies sind Themen, die durch eine Ethik-Infrastruktur erfasst werden. In allen Fällen geht es um Patientenwohl und Gefährdung, um Würde, Gerechtigkeit, Autonomie. Und in sehr vielen Fällen erfolgt die Beseiti- 
gung solcher Missstände nicht durch kluge Philosophie, sondern durch ein waches und konsequentes Management.

\subsection{Interkultureller und interreligiöser Respekt}

In Zeiten der Globalisierung und eines wachsenden Bevölkerungsanteils mit Migrationshintergrund begegnen sich Menschen mit unterschiedlichsten kulturellen und religiösen Mustern. Diese Muster spielen im Zusammenhang von Gesundheit und Krankheit, Sexualität, Geburt, Sterben und Tod eine sehr große Rolle. Mit Unterschieden in den Geschlechtsrollen und den Familienstrukturen unterscheiden sich auch die Entscheidungssubjekte interkulturell. In deutschen Krankenhäusern stammen nicht nur die Patienten zunehmend aus verschiedensten Kulturkreisen, gleiches gilt auch für die Mitarbeiter.

Die respektvolle Wahrnehmung der jeweiligen Werte der Individuen und ihrer Familien ist eine wichtige Voraussetzung für eine gute Versorgungsqualität. Ethische Konflikte können auftreten, wenn die Besonderheiten einer Kultur mit den ethischen und rechtlichen Grundlagen der deutschen und europäischen Krankenhausmedizin in Konflikt geraten.

So kann das ethische Konzept der Autonomie, eines der wichtigsten „Biomedical Principles“ der modernen Medizin- und Bioethik, nicht einfach als universal vorausgesetzt werden (s. Kap. 2). In vielen Kulturen entscheidet nicht das jeweilige Individuum über seine medizinischen Geschicke, sondern die Familie oder eine andere soziale Einheit. Auch ist das Sprechen über Sterben und Tod nicht in allen Kulturen gleich bewertet und erlaubt. Bei klinischen Entscheidungen stehen die Behandlungsteams häufig im Konflikt, an welchen kulturellen Werten sie sich orientieren sollen. Der hierbei unterstützende Wert ethisch reflektierter Entscheidungsprozesse und der sie fördernden Ethik-Infrastruktur ist naheliegend.

\section{Coma vigile}

Auf der Intensivstation einer Universitätsklinik lag eine junge Frau im Wachkoma. Ihre Großfamilie stammt aus Afghanistan. Geboren wurde sie in Deutschland. Aufgrund ihrer schweren chronischen Erkrankung hatte sie diesen Zustand vorausgesehen und eine Patientenverfügung verfasst, die präzise ihre jetzige Situation beschrieb. Sie wollte keine lebensverlängernden Maßnahmen. Das Behandlungsteam sah sich mit dem Familienoberhaupt, dem über 90-jährigen Großvater der Frau konfrontiert. Dieser sah sich selbstverständlich in der Rolle des Entscheiders. Mit Hilfe eines Dolmetschers forderte er eine unbegrenzte Fortsetzung lebenserhaltender Maßnahmen. In seinen Augen 
schlief seine Nichte nur und sie würde zu gegebener Zeit wieder aufwachen. Das medizinische Team folgte seinen Forderungen. Nach deutschen Maßstäben war dies ethisch und rechtlich nicht akzeptabel. Erst eine ethische Fallbesprechung trug dazu bei, den Willen der Patientin nach Abbruch lebenserhaltender Maßnahmen zu befolgen.

Aus organisationsethischer Sicht sprechen viele Cründe für die Aufnahme einer expliziten, durchdachten Vorstellung von Interkulturalität in das Leitbild der Einrichtungen. Auch hier bewährt sich eine Unternehmenskultur, die der Herausforderung Interkulturalität mit Sensibilität, ethischer Bewusstheit und Professionalität begegnet.

\subsection{Misserfolg: Halbherzige Ethik}

Wenn die Aufnahme von Ethik-Projekten sich beschränkt auf die Verkündung von Vorsätzen, wenn Leitbilder ethische Prinzipien enthalten, die in der Praxis des Unternehmens nicht effektiv umgesetzt werden, wenn mit Ethik-Strukturen lediglich Kriterien für Zertifizierungen erfüllt werden ohne dass diese effektiv in die Entscheidungsprozesse integriert sind, dann besteht die sehr reale Gefahr zynischer Abwehr durch die Mitarbeiterschaft.

Unternehmensleitbilder von Krankenhausunternehmen werden von zu vielen Ärzten, Pflegenden, Therapeuten und Dienstleistern als unehrlich, unwichtig oder gar als zynisch wahrgenommen. Als Mitarbeiter eines großen Klinikums zu Beginn eines Ethik-Projekts danach befragt wurden, was sie sich denn davon erhofften, war die Antwort der großen Mehrheit, sie wünschten sich ein Ende der Propagandasprüche, die eine heile Welt vortäuschten, die mit er Wirklichkeit in ihrem Haus nichts zu tun hätten. Als eines der ersten Ergebnisse des Projekts bemühte sich die Klinikleitung fortan um mehr Ehrlichkeit in der Unternehmenskommunikation und in der Darstellung nach außen.

Wenn Ethik zu leeren Propagandasprüchen verkommt, die eine Wirklichkeit vorspiegeln möchte, die es so nicht gibt, dann ergibt sich ein Risiko für das ganze Krankenhaus. Ethische Unternehmensgrundsätze müssen ehrlich befolgt werden, andernfalls führen sie zu zynischen Abwertungen, die dem Ruf des Hauses abträglich sind.

In einem anderen Klinikum wurden die Mitarbeiter danach befragt, ob sie sich im Falle eines Konflikts auf die Ethik-Leitlinien des Hauses bezie- 
hen könnten. Das Ergebnis war ernüchternd. Alle Befragten antworteten mit Nein. Man hatte es bei den Ethik-Leitlinien belassen und nicht weiter an der Entwicklung der „Corporate Ethical Identity“ gearbeitet.

Wenn ein Klinikunternehmen einen hohen ethischen Anspruch verfolgt, der immer ein Kernelement jedes Qualitätsversprechens ist, dann muss effektiv die Beachtung oder Missachtung dieser Leitlinien kontrolliert werden. Das muss an der Spitze des Unternehmens beginnen und alle Prozesse umfassen, von der ärztlichen Praxis bis zur Logistik oder der Küche. Vorbildlich ist hier das Modell des Kommunalunternehmens Klinikum Nürnberg, das die Mitarbeiter ausdrücklich ermuntert, Verletzungen ethischer Positionen zur Sprache zu bringen zwecks Erhöhung der Qualität aller Dienstleistungen, allen voran der Medizin und Pflege.

Viele Kliniken, die sich für die Einrichtung von Klinischen Ethik-Komitees entschieden haben, haben deren Auftrag auf ethische Fallbesprechungen zu patientenbezogenen medizinischen Entscheidungen begrenzt. Oft konnte beobachtet werden, dass die Komitees darauf warteten, ab und an einen „Fall“ vorgelegt zu bekommen, den man diskutierte und zu dem eine Empfehlung abgegeben wurde. Ein proaktives Engagement wurde diesen Komitees nicht erlaubt, organisationsethische Themen wurden ausgeschlossen. Diejenigen Mitarbeiter, die direkt in der Patientenversorgung tätig waren, fand man hier selten. Dementsprechend war das Ansehen des Komitees gering. Es wurde der Feigenblattfunktion verdächtigt.

Die Ernsthaftigkeit eines Ethik-Projekts kann daran bemessen werden, ob neben Praktikern aus Medizin, Pflege und Seelsorge auch Angehörige des Managements und der Verwaltungsberufe dabei sind. Ohne direkte Verbindung zwischen Ethik-Struktur und der Leitungsebene bleiben viele dort geführte Diskussionen belanglos.

Die größte Gefahr halbherziger oder gar nur propagandistischer EthikProjekte besteht in der damit einhergehenden Zersetzung von Vertrauen. Ethik darf nicht dazu dienen, Defizite der Patientenversorgung und der Mitarbeiterführung zu kaschieren. Im Gegenteil. Es geht um die fortwährende Arbeit an hoher Versorgungsqualität, die durch komplexe arbeitsteilige Teams geleistet werden muss.

\subsection{Ethik als ultima ratio}

Wenn hoher medizinischer Einsatz für den Patienten nicht genügend von den Finanzierungsregeln für Krankenhäuser unterstützt wird, wenn die sorgfältige Betreuung eines Patienten zu wirtschaftlichen Verlusten für 
ein Krankenhaus führt, wenn sich eine Intensivstation oder Notaufnahme „nicht rechnet“, dann sind Konflikte zwischen „Ethik und Ökonomie“ vorprogrammiert. Dann kann sich die kaufmännische Leitung gezwungen sehen, das wirtschaftliche Ergebnis des Hauses, damit ggf. auch sein Überleben, zu Lasten der medizinischen Qualität in den Vordergrund zu stellen. Dies soll nach Möglichkeit vermieden werden und wenn es doch stattfindet wird es häufig geleugnet. Das Management befindet sich hier in einer schwierigen Situation. Schon aus Gründen des Wettbewerbs kann es sich nicht leisten, Abstriche an der Qualität zuzugeben. Es hat auch nicht die Möglichkeit, durch politische Aktivitäten die Regeln der Krankenhausfinanzierung zu ändern. Selbst wenn es über die Arbeit der Krankenhausverbände und Kammern zu Verbesserungen kommt, brauchen diese Entwicklungen Zeit. Solange muss innerhalb des Klinikums nach Kompromissen gesucht werden. Der Erhalt eines von der Insolvenz bedrohten Hauses mit den daran hängenden Arbeitsplätzen und dem Versorgungangebot kann ein ebenso wertvolles Ziel sein wie die Einhaltung höchster Sicherheitsstandards für Patienten. Individualisierte, auf $\mathrm{Pa}$ tienten bezogene Medizinethik und eine Ethik der Bevölkerungsgesundheit („Public Health Ethik“) stehen sich gegenüber. Erkennt man allein die Medizinethik als gültige Orientierung im Krankenhaus an so wird die Problematik des Erhalts der Einrichtungen des Gesundheitssystems nicht hinreichend gewürdigt.

Innerhalb der Kliniken kann in einer solchen Situation nur gehofft werden, dass die Vertreter von Qualität und Wirtschaftlichkeit im offenen Dialog miteinander stehen und dass für beide Aspekte genügend Anerkennung gefunden wird. Hierfür ist Ethik-Kompetenz essentiell. Gerade in solchen schwierigen wirtschaftlichen Situationen ist Ethik also wichtig und kann die Unternehmenskultur am Leben erhalten, um so gemeinsam Kompromisse und Lösungen zu erarbeiten und nicht noch zusätzliche Ressourcen in innerbetrieblichen Konflikten zu verschwenden.

\subsection{Ethik und wirtschaftlicher Erfolg}

„Ethik? Das können wir uns nicht leisten, das ist nur etwas für Krankenhäuser, die bereits wirtschaftlich erfolgreich sind. Vielleicht machen wir das später einmal, wenn wir schwarze Zahlen schreiben ..." (Aussage eines kaufmännischen Geschäftsführers eines kommunalen Krankenhauses, 2015)

"Organizations are no longer built on force but on trust. The existence of trust between people does not necessarily mean that they like one another. It means that they understand one another. Taking responsibility for relationships is therefore an absolute necessity. It is a duty. [...]" (Drucker 1999) 
Kliniken, die über ein Ethik-Management und eine Ethik-Infrastruktur verfügen sind in der Regel überwiegend wirtschaftlich erfolgreich, auch unter schwierigen Bedingungen. Damit soll nicht gesagt sein, dass „Ethik“ allein für diese Erfolge verantwortlich ist. Es wäre auch denkbar, dass sich tatsächlich nur wirtschaftlich erfolgreiche Krankenhäuser eine Ethik-Infrastruktur leisten und dies somit nicht Ursache, sondern Folge des wirtschaftlichen Erfolges wäre. Es gibt jedoch viele Cründe, die für die Annahme sprechen, dass ehrliche Ethik-Projekte den wirtschaftlichen Erfolg unterstützen. Denkbar ist freilich auch, dass erfolgreiches Management offener ist für die Bedeutung der Unternehmenskultur und der darin enthaltenen ethischen Aspekte ist.

Unabhängig vom Wunsch der Patienten, Mitarbeiter und der Gesellschaft nach hohen qualitativen und ethischen Standards der medizinischen Versorgung, spielt doch die Wirtschaftlichkeit bzw. Rentabilität der Krankenhäuser in fast allen Gesundheitssystemen eine entscheidende Rolle. Die Ursachen hierfür sind vielfältig, basieren aber im Wesentlichen auf ordnungspolitischen Vorgaben und einer weitreichenden Ressourcenknappheit, die sich nicht nur auf Geld, sondern zunehmend auch auf Personal erstreckt. Prinzipiell kann das Streben nach Rentabilität dabei zu unterschiedlichen Ergebnissen führen. Zum einen kann sich eine Verbesserung der Effizienz bei gleichbleibender oder sogar steigender Qualität ergeben (dies ist der Fall, wenn Prozesse effizienter organisiert werden, beispielsweise durch ein Neubauprojekt). Zum anderen kann die Verbesserung der Effizienz zum Preis einer Qualitätsreduktion herbeigeführt werden (dies kann der Fall sein, wenn eine Personalreduktion zu deutlichen Kosteneinsparungen bei stabilen Fallzahlen führt aber gleichzeitig die Ergebnisqualität sinkt). Vielfach lässt sich in der Praxis eine Mischung beider Wirkmechanismen beobachten. Das bedeutet, es werden teils Bemühungen um eine Verbesserung der Qualität und Effizienz durchgeführt, teilweise aber auch Sparmaßnahmen oder Erlösoptimierungen, die durch Über-, Unter- oder Fehlversorgung letztlich die Qualität reduzieren. Aufgrund des systemseitig gegebenen starken wirtschaftlichen Druckes ist es nicht selten für die Krankenhausleitung schwierig, hier eine saubere Trennung vorzunehmen.

Wenn ein hoher Einfluss ethischer Werte auf die medizinischen und wirtschaftlichen Entscheidungen im Krankenhaus gefördert wird, so ist es wahrscheinlich, dass Maßnahmen zur Einsparung qualitätsrelevanter Ressourcen (z.B. Personalstärke) sowie Anreize zur erlössteigernden Übertherapie weniger häufig ergriffen werden. Auf diesen Ausschnitt bezogen, wäre Ethik wirtschaftlich kontraproduktiv. Der wirtschaftliche Erfolg eines Krankenhauses ist aber von mehr Faktoren abhängig und so steht dem gegenüber der Nutzen eines ethischen Managements und ethischer 
Medizin auf verschiedenen anderen Ebenen. Auch wenn es für das Krankenhausmanagement schwer vorherzusagen ist, ob sich die Investition in Ethik auch betriebswirtschaftlich lohnt, so zeigen doch sowohl die Erfahrungen im Rahmen diverser Ethik-Projekte, als auch wirtschaftspsychologische und wirtschaftsethische Studien in anderen Branchen, dass Ethik einen Beitrag für einen nachhaltigen betriebswirtschaftlichen Erfolg im Krankenhaus leisten kann.

Im Vordergrund stehen dabei die oben beschriebenen positiven Auswirkungen auf die direkte medizinische Qualität. Hieraus kann sich zudem eine bessere Interdisziplinarität und Kollegialität, nicht nur zwischen den medizinischen Fachdisziplinen, sondern auch zwischen der Medizin und dem Management sowie den anderen Berufsgruppen ergeben. Die Folge ist eine signifikante Verbesserung der Mitarbeiterzufriedenheit mit geringerer Fluktuation und höherer Arbeitgeberattraktivität. Dieser Aspekt bietet vor dem Hintergrund des Fachkräftemangels einen direkten wirtschaftlichen Vorteil, genauso wo die Strahlwirkung auf Zuweiser und Patienten. Ein weiterer direkter wirtschaftlicher Vorteil ist von der verbesserten Identifikation der Mitarbeiter mit den ganzheitlichen Zielen des Krankenhauses zu erwarten, der einen Beitrag zu einem effizienten Ressourceneinsatz führen kann (Vermeidung von Verschwendung durch Übernahme von Verantwortung). Als Nebeneffekt der gemeinsamen Zielausrichtung und Identifikation mit dem Krankenhaus werden sich zudem Bonuszahlungen reduzieren lassen, die in der Regel ohnehin nur bedingt wirtschaftlich sinnvoll sind.

\section{Direkte und indirekte Auswirkungen von Ethik auf den wirtschaftlichen Erfolg eines Krankenhauses:}

- Verbesserung der medizinischen Qualität

- Verbesserung der Interdisziplinarität und Kollegialität

- Verbesserung der Mitarbeitermotivation

- Erhöhte Arbeitsplatzattraktivität, Reduktion der Mitarbeiterfluktuation

- Reduktion von variablen Vergütungsanteilen zur Mitarbeiterbindung

- Verbesserung der Patientenzufriedenheit

- Verbesserung der Zuweiserzufriedenheit

- Erhöhte Identifikation der Mitarbeiter mit Unternehmenszielen

- Erhöhte Effektivität der Arbeitsleistung

- Geringere Verschwendung und höhere Effizienz

Ob sich diese positiven Effekte im jedem Krankenhaus erreichen lassen und welcher tatsächliche Einfluss sich auf die betriebswirtschaftliche Bi- 
lanz ergibt, wird sich in der Regel erst mittelfristig zeigen und auch nicht immer eindeutig zuzuordnen lassen. Insbesondere Über-/Unter- und Fehlversorgung werden allerdings maßgeblich durch eine ausgeprägte Sorge um Qualität und Ethik minimiert werden, wodurch es teilweise zu Mindererlösen und verstärkter Ressourcenbelastung kommen kann. Dieser Effekt sollte sich aber durch die oben beschriebenen positiven Effekte ausgleichen lassen. Für den nachhaltigen Erfolg des Krankenhauses erscheint es also plausibel, dass die Investition in Ethik sowohl für die Verbesserung der Gesamtqualität als auch für den langfristigen betriebswirtschaftlichen Erfolg wertschöpfend ist. Neben der ohnehin gebotenen moralischen Verpflichtung ein Krankenhaus ethisch zu führen, gibt es hierfür also auch gewichtige wirtschaftliche Gründe.

\section{Weiterführende Literatur}

Marckmann G, Maschmann I (2014) Zahlt sich Ethik aus? Notwendigkeit und Perspektiven des Wertemanagements im Krankenhaus. Z Evid Fortbild Qual Gesundhwes 2014, 108 (2-3):157-65

Naegler H (2011) Management der sozialen Verantwortung im Krankenhaus. Medizinisch Wissenschaftliche Verlagsgesellschaft Berlin

Sprenger RK (2015) Das anständige Unternehmen: Was richtige Führung ausmacht - und was sie weglässt. 2. Auflage. Deutsche Verlags-Anstalt München

Peus C, Kerschreiter R, Frey D, Traut-Mattausch E (2010) What is the value? Economic Effects of Ethically-Oriented Leadership. Journal of Psychology, 218 (4):198-212

\section{Zitierte Literatur}

Drucker PF (1999) Managing oneself. Harvard Business Review

Reinhard R, Vasek T (2015) R. Sprenger interviewt von Rebekka Reinhard und Thomas Vasek. In: Hohe Luft spezial Führung, Beilage Handelsblatt, 2016, mit Verweis auf Reinhard K. Sprenger, Das anständige Unternehmen, DVA, 2015:18-22 